\title{
Rumo à educação baseada em competências: construindo a matriz do internato em Medicina de Família e Comunidade
}

\author{
Towards competency-based education: building the Family Medicine clerkship blueprint \\ Hacia una educación basada en competencias: la construcción de la matriz de prácticas en \\ Medicina de Familia \\ Ângela Jornada Benª, José Mauro Ceratti Lopesa ${ }^{\mathrm{a}}$, Carmen Vera Giacobbo Daudta , Maria Eugênia Bresolin Pintoa ${ }^{\mathrm{a}}$, Mônica Maria \\ Celestina de Oliveira ${ }^{a}$
}

\section{Resumo}

Objetivo: Elaborar a matriz de competências a serem desenvolvidas pelos estudantes durante os três meses de estágio no internato em Medicina de Família e Comunidade na Universidade Federal de Ciências da Saúde de Porto Alegre (UFCSPA). Métodos: Foi realizada revisão de literatura sobre o referencial teórico do ensino baseado em competências sobre escolas médicas que utilizassem o ensino baseado em competências na especialidade e sobre as principais diretrizes nacionais e internacionais sobre o tema. Diante da revisão de literatura, foi discutida a aplicabilidade das recomendações no contexto do internato da UFCSPA. Resultados: Dezoito competências foram elaboradas e divididas em quatro eixos: Geral; Abordagem Individual; Abordagem Familiar e Abordagem Comunitária. Cada competência foi detalhada em um ou mais componentes. Para cada componente, foram identificados os métodos de ensino e de avaliação atualmente utilizados no programa do internato. Conclusão: Com a definição da matriz, as competências, antes desenvolvidas de forma intuitiva, foram organizadas de modo a viabilizar a revisão e adequação dos processos de ensino e avaliação.

\section{Abstract}

Objective: To elaborate a blueprint of the competencies to be developed during three months of Family Medicine Clerkship at the Federal University of Health Sciences of Porto Alegre (UFCSPA). Methods: A literature review was carried out on competency-based education, medical schools using this approach and national and international guidelines on medical education. Therefore, the applicability of the recommendations in the context of UFCSPA Clerkship was discussed. Results: Eighteen competencies were established and divided into four axes: General, Individual Approach, Family Approach, and Community Approach. Each competency was depicted in one or more components. For each component, we have identified the teaching and students' assessment methods currently applied in Family Medicine Clerkship. Conclusions: Clerkship competencies, which were previously developed in an intuitive way, were organized in a blueprint that enables the review and adequacy of teaching methods and students' assessment.
Palavras-chave: Educação Baseada em Competências Educação Médica Internato e Residência Medicina de Família e Comunidade

Keywords: Competency-Based Education Medical Education Internship and Residency Family Practice
Como citar: Ben AJ, Lopes JMC, Daudt CG, Pinto MEB, Oliveira MMC. Rumo à educação baseada em competências: construindo a matriz do internato em Medicina de Família e Comunidade. Rev Bras Med Fam Comunidade. 2017;12(39):1-16. http://dx.doi.org/10.5712/rbmfc12(39)1354
Fonte de financiamento: declaram não haver.

Parecer CEP: 837.591 (UFCSPA), aprovado em 20/10/2014.

Conflito de interesses: declaram não haver. Procedência e revisão por pares: revisado por pares. Recebido em: 16/07/2016. Aprovado em: 22/04/2017. 


\section{Resumen}

Objetivo: Elaborar una matriz de competencias, que debe ser desarrollada durante tres meses de Internado de Medicina Familiar y Comunitaria en la Universidad Federal de Ciencias de la Salud de Porto Alegre (UFCSPA). Métodos: Se realizó una revisión de la literatura sobre el marco teórico de la educación basada en competencias sobre las escuelas de medicina que utilizara la educación basada en competencias en la especialidad y sobre las principales directrices nacionales e internacionales sobre el tema. Por lo tanto, se discutió la aplicabilidad de las recomendaciones en el contexto del internado de UFCSPA. Resultados: Se elaboraron dieciocho competencias y se dividieron en cuatro ejes: General, Enfoque Individual, Enfoque Familiar y Enfoque Comunitario. Cada competencia se representó en uno o más componentes. Para cada componente se identificaron los métodos de enseñanza y de evaluación actualmente utilizados en el programa del internado. Conclusiones: Las competencias del Internado, antes desarrolladas de manera intuitiva, fueron organizadas en una matriz para hacer posible la revisión y adecuación de los métodos de enseñanza y la evaluación.

\section{Introdução}

O ensino por competências tem sido adotado por escolas médicas com o objetivo de orientar a formação baseada na aplicação do conhecimento e no desenvolvimento de habilidades e atitudes. Esse método pressupõe uma avaliação formativa, ou seja, que se avalie o desempenho do estudante em relação às competências previamente estabelecidas. ${ }^{1,2}$ Tradicionalmente, o ensino médico está fundamentado na aquisição de conhecimentos com exposição a conteúdos médicos específicos distribuídos em disciplinas.

Os métodos de ensino são, geralmente, baseados em aulas expositivas e o processo de aprendizagem é avaliado de forma somativa, ${ }^{3}$ medindo, principalmente, a capacidade de memorização e aquisição de conhecimento. O modelo tradicional tem sido questionado por apresentar limitações quanto à capacidade de formar profissionais aptos a responderem às demandas sociais atuais, como ter habilidades comunicacionais e culturais ou capacidade para resolver problemas que vão além do conhecimento teórico. ${ }^{4,5}$

Entende-se por competência a capacidade de mobilizar e integrar conhecimentos, habilidades e atitudes para resolver problemas num contexto profissional específico. ${ }^{6}$ As Diretrizes Curriculares Nacionais do Curso de Graduação em Medicina (DCNs) incorporaram esse conceito e recomendam que as Instituições de Ensino Superior organizem, desenvolvam e avaliem a formação médica baseada nesse modelo, a fim de contemplar as necessidades do Sistema Único de Saúde (SUS).

Há também uma demanda crescente por profissionais médicos, principalmente após a adoção da Estratégia de Saúde da Família como política de expansão e qualificação do acesso aos serviços de saúde pela população. Por outro lado, os resultados do estudo "Demografia Médica no Brasil 2015"7 mostram grande número de práticas profissionais não padronizadas e concentração de profissionais em especialidades médicas não diretamente ligadas à atenção primária. Isso pode ser entendido como um processo de formação não alinhado com os princípios do Sistema Nacional de Saúde.

Assim, o objetivo desse artigo é apresentar a Matriz de Competências desenvolvida para o internato em Medicina de Família e Comunidade no contexto de uma Instituição de Ensino Superior que utiliza o modelo tradicional de ensino a fim de iniciar o processo de incorporação do ensino baseado em competências ao currículo.

\section{Métodos}

Foi realizada revisão de literatura sobre o referencial teórico do ensino baseado em competências, sobre escolas médicas que utilizassem o ensino baseado em competências na especialidade e sobre as principais recomendações das entidades nacionais e internacionais envolvidas com o ensino médico. 
O critério de seleção foi encontrar definições de competências para o exercício profissional e modelos de matriz de competências utilizados. As bases de dados consultadas foram PubMed, Scielo e Google Acadêmico. Foi utilizada uma combinação de termos de busca amplos em português, inglês e espanhol como Educação Baseada em Competências, Competency-Based Education, Educación Basada en Competencias, Medicina de Família e Comunidade, Family Medicine e Medicina Familiar y Comunitaria, Internato, Clerkship, Internship, Internado, matriz de competências, blueprint, competência, competency. Sites de escolas médicas e recomendações nacionais e internacionais foram acessados conforme referências indicadas em artigos, livros e ou por colegas docentes de outras escolas médicas.

O processo de construção da Matriz foi iniciado no curso de desenvolvimento docente na Universidade Federal de Ciências da Saúde de Porto Alegre (UFCSPA) sob coordenação dos professores do Núcleo de Apoio Pedagógico em 2012 e no curso Leonardo níveis I e II da European Academy of Teachers of General Practice and Family Medicine (EURACT) para formadores em MFC em 2013. As etapas seguintes foram realizadas em 2014 e 2015 como parte do projeto desenvolvido no programa de desenvolvimento docente da Foundation for Advancement of International Medical Education - Instituto Regional FAIMER - Brasil, reconhecido como especialização pela Universidade Federal do Ceará.

\section{Resultados}

A revisão de literatura sobre o referencial teórico do ensino baseado em competências mostrou que várias escolas médicas na Europa e América do Norte utilizam essa metodologia em seus currículos e que, essencialmente, seguem as recomendações de intituições acreditadoras. ${ }^{1,2,4,6,8-15}$ Tendo em vista o grande número de escolas, optou-se por consultar os sites das instituições acreditadoras reiteradamente citadas nas referências consultadas, com objetivo de encontrar, primeiramente, a definição das competências para o exercício profissional.

São elas: Association for Medical Education in Europe (AMEE), ${ }^{16}$ Accreditation Council for Graduate Medical Education (ACGME), ${ }^{17}$ Association of American Medical Colleges ${ }^{18}$ e Canadian Medical Education Family Medicine (CanMEDS-FM). ${ }^{19}$ Foram encontrados estudos sobre definição de competências para residência médica e diretrizes com objetivo de incentivar a incorporação do ensino por competências nos currículos da graduação e integração com os da residência. ${ }^{20}$

Na América Latina ${ }^{11,12,21,22}$ e no Brasil, algumas escolas implementaram o currículo por competências. Foram encontradas referências sobre três escolas médicas brasileiras, a Universidade Estadual de Londrina (UEL), ${ }^{14}$ Universidade da Cidade de São Paulo (UNICID) ${ }^{9}$ e Faculdade de Medicina de Marília (FAMEMA). ${ }^{15}$ Os sites dessas Instituições apresentam informações sobre perfil geral do egresso e sobre competências recomendadas nas Diretrizes Curriculares Nacionais do Curso de Graduação em Medicina (DCNs), não sendo informado sobre matriz de competências adaptadas ao contexto local.

Como não foram encontrados modelos específicos de matriz de competências para graduação e, principalmente, para o internato em MFC ao nível nacional, optou-se por selecionar as recomendações da $\mathrm{DCNs}^{23}$ e da Sociedade Brasileira de Medicina de Família e Comunidade para a residência médica (SBMFC). ${ }^{24}$ 
A fim de complementar as competências gerais requeridas para o exercício profissional das DCNs e da SBMFC, foram também selecionadas duas recomendações internacionais por serem reiteradamente citadas nas referências consultadas: Canadian Medical Education - Family Medicine (CanMEDS-FM); ${ }^{19}$ e Family and Community Medicine - University of Toronto. ${ }^{25}$ No site da Universidade de Toronto foram encontradas descritas as competências principais para o interno em MFC. Assim, foi realizada adaptação dos modelos das DCNs, SBMFC, CanMEDS-FM e da Universidade de Toronto ao contexto do internato em MFC em nossa Instituição.

Adaptando tais recomendações ao contexto do internato, foi definida uma Matriz detalhando 18 competências a serem desenvolvidas pelo interno durante os 3 meses de estágio apresentadas no Quadro 1. As competências foram organizadas em quatro eixos: Geral, Abordagem Individual, Abordagem Familiar e Abordagem Comunitária. Um eixo pode conter uma ou mais competências. Uma competência pode abranger um ou mais componentes. Para cada componente, foram identificados os métodos de ensino e avaliação atualmente utilizados no programa do internato apresentadas nos Quadros 2, 3, 4 e 5. O conjunto de competências dispostas nos quatro eixos compõe o perfil esperado do estudante que concluiu o estágio na especialidade de Medicina de Família e Comunidade.

Quadro 1. Matriz de Competências do Internato em Medicina de Família e Comunidade conforme eixos.

\begin{tabular}{|c|c|}
\hline EIXO & COMPETÊNCIAS \\
\hline \multirow{3}{*}{ GERAL } & (1) Demonstrar consciência da necessidade de ser um eterno aprendiz. \\
\hline & (2) Demonstrar habilidades de comunicação efetiva, profissional e sem preconceitos. \\
\hline & (3) Entender o papel do Médico de Família e Comunidade no sistema de saúde. \\
\hline \multirow{13}{*}{ INDIVIDUAL } & (4) Adotar abordagem centrada na pessoa. \\
\hline & (5) Realizar anamnese e exame físico de forma apropriada para o internato. \\
\hline & (6) Elaborar lista de diagnóstico diferencial condizente com os dados coletados na anamnese e exame físico. \\
\hline & (7) Reconhecer as apresentações típica e atípica das doenças prevalentes na APS e das doenças com risco de morte. \\
\hline & (8) Demonstrar uma abordagem eficaz para a apresentação de sintomas sem explicação médica. \\
\hline & $\begin{array}{l}\text { (9) Demonstrar uma abordagem eficaz para a apresentação de doença aguda autolimitada e doença } \\
\text { potencialmente fatal. }\end{array}$ \\
\hline & (10) Demonstrar uma abordagem eficaz em relação às doenças crônicas. \\
\hline & (11) Demonstrar uma abordagem eficaz em relação às doenças com um forte componente emocional/saúde mental. \\
\hline & $\begin{array}{l}\text { (12) Modificar o diagnóstico diferencial tendo em vista sintomas inesperados ou modificados, ou quando os } \\
\text { sintomas persistem além do esperado. }\end{array}$ \\
\hline & (13) Justificar escolha de exames laboratoriais e utilizá-los apenas quando houver impacto no manejo do paciente. \\
\hline & (14) Interpretar os testes diagnósticos pronta e adequadamente. \\
\hline & (15) Comunicar os resultados em tempo hábil. \\
\hline & (16) Desenvolver um plano de tratamento adequado. \\
\hline FAMILIAR & (17) Adotar abordagem centrada na pessoa considerando contexto familiar. \\
\hline COMUNITÁRIA & (18) Envolver outros recursos de forma adequada no sistema de saúde. \\
\hline
\end{tabular}


Quadro 2. Matriz de Competências do Internato em Medicina de Família e Comunidade no Eixo Geral.

\begin{tabular}{|c|c|c|c|c|}
\hline EIXO & COMPETÊNCIAS & COMPONENTES & MÉTODOS DE APRENDIZADO & MÉTODOS DE AVALIAÇÃO \\
\hline \multirow{9}{*}{ GERAL } & $\begin{array}{l}\text { Demonstrar consciência } \\
\text { da necessidade de ser } \\
\text { um eterno aprendiz. }\end{array}$ & $\begin{array}{l}\text { Aplicar, sempre que possível, } \\
\text { os princípios da medicina } \\
\text { baseada em evidências. } \\
\text { Comprometer-se com o } \\
\text { acompanhamento dos } \\
\text { casos clínicos, a fim de } \\
\text { aprender com os desfechos } \\
\text { favoráveis e desfavoráveis } \\
\text { dos pacientes. }\end{array}$ & $\begin{array}{l}\text { Discussão de casos clínicos. } \\
\text { Exemplo do preceptor e do } \\
\text { professor especialista em MFC. } \\
\text { Dramatização. } \\
\text { Discussão de casos clínicos. } \\
\text { Exemplo do preceptor e do } \\
\text { professor especialista em MFC. } \\
\text { Dramatização. }\end{array}$ & $\begin{array}{l}\text { Observação direta } \\
\text { Vídeogravação + Relatório } \\
\text { da consulta filmada }\end{array}$ \\
\hline & & $\begin{array}{l}\text { Utilizar diferentes técnicas } \\
\text { de comunicação: realizar } \\
\text { perguntas abertas e } \\
\text { fechadas, questões diretas, } \\
\text { escalas e narrativas. }\end{array}$ & $\begin{array}{l}\text { Discussão de casos clínicos. } \\
\text { Exemplo do preceptor e do } \\
\text { professor especialista em MFC. } \\
\text { Dramatização. }\end{array}$ & $\begin{array}{l}\text { Observação direta } \\
\text { Vídeogravação + Relatório } \\
\text { de autoavaliação da } \\
\text { consulta filmada }\end{array}$ \\
\hline & $\begin{array}{l}\text { Demonstrar habilidades } \\
\text { de comunicação efetiva, } \\
\text { profissional e sem } \\
\text { preconceitos }\end{array}$ & $\begin{array}{l}\text { Utilizar estilo flexível que se } \\
\text { adapte a várias culturas e } \\
\text { níveis escolares (princípio da } \\
\text { competência cultural). }\end{array}$ & $\begin{array}{l}\text { Discussão de casos clínicos. } \\
\text { Exemplo do preceptor e do } \\
\text { professor especialista em MFC. } \\
\text { Dramatização. }\end{array}$ & $\begin{array}{l}\text { Observação direta } \\
\text { Vídeogravação }\end{array}$ \\
\hline & & $\begin{array}{l}\text { Demonstrar abordagem } \\
\text { apropriada para idade do } \\
\text { paciente (ex. jogos e humor } \\
\text { com crianças, fala mais clara } \\
\text { com idosos). }\end{array}$ & $\begin{array}{l}\text { Discussão de casos clínicos. } \\
\text { Exemplo do preceptor e do } \\
\text { professor especialista em MFC. } \\
\text { Dramatização. }\end{array}$ & $\begin{array}{l}\text { Observação direta } \\
\text { Vídeogravação }\end{array}$ \\
\hline & \multirow{5}{*}{$\begin{array}{l}\text { Entender o papel do } \\
\text { MFC no sistema de } \\
\text { saúde. }\end{array}$} & $\begin{array}{l}\text { Caracterizar como o } \\
\text { conhecimento, habilidades } \\
\text { e atitudes do MFC o } \\
\text { diferenciam dos demais } \\
\text { especialistas. }\end{array}$ & $\begin{array}{l}\text { *Estudo de texto: } \\
\text { Capítulo 1. Princípios da Medicina de } \\
\text { Família e Comunidade. } \\
\text { Discussão de casos clínicos. }\end{array}$ & $\begin{array}{l}\text { Teste de conhecimento } \\
\text { aplicado } \\
\text { Observação direta } \\
\text { Vídeogravação }\end{array}$ \\
\hline & & $\begin{array}{l}\text { Proporcionar cuidado } \\
\text { continuado. }\end{array}$ & $\begin{array}{l}\text { Organização da agenda na unidade } \\
\text { de saúde para garantir consultas de } \\
\text { retorno. }\end{array}$ & $\begin{array}{l}\text { Observação direta } \\
\text { Vídeogravação }\end{array}$ \\
\hline & & $\begin{array}{l}\text { Utilizar os princípios do MFC } \\
\text { embasado no conhecimento } \\
\text { do ciclo de vida e nos } \\
\text { atributos da APS. }\end{array}$ & $\begin{array}{l}\text { *Estudo de texto: } \\
\text { Capítulo 4. Atenção Primária à } \\
\text { Saúde no Brasil. } \\
\text { Ciclo de vida PROMEF } \\
\text { Discussão de casos clínicos } \\
\text { Tarefas: teste }\end{array}$ & $\begin{array}{l}\text { Teste de conhecimento } \\
\text { aplicado } \\
\text { Observação direta } \\
\text { Vídeogravação }\end{array}$ \\
\hline & & $\begin{array}{l}\text { Proporcionar cuidado } \\
\text { preventivo adequado. }\end{array}$ & $\begin{array}{l}\text { *Estudo de texto: } \\
\text { Capítulo 61. Rastreamento de } \\
\text { doenças. } \\
\text { Capítulo 74. Saúde da Criança. } \\
\text { Capítulo 75. Saúde do Homem. } \\
\text { Capítulo 76. Saúde da Mulher. } \\
\text { Capítulo 77. Saúde do Idoso. } \\
\text { Discussão de casos clínicos }\end{array}$ & $\begin{array}{l}\text { Teste de conhecimento } \\
\text { aplicado } \\
\text { Observação direta } \\
\text { Vídeogravação }\end{array}$ \\
\hline & & $\begin{array}{l}\text { Entender o papel do MFC } \\
\text { na promoção de saúde das } \\
\text { comunidades. }\end{array}$ & $\begin{array}{l}\text { *Estudo de texto: } \\
\text { Capítulo } 6 \text {. Cultura, Saúde e o MFC. } \\
\text { Discussão de casos clínicos }\end{array}$ & $\begin{array}{l}\text { Teste de conhecimento } \\
\text { aplicado } \\
\text { Observação direta } \\
\text { Vídeogravação }\end{array}$ \\
\hline
\end{tabular}


Quadro 3. Matriz de Competências do Internato em Medicina de Família e Comunidade no Eixo Abordagem Individual.

\begin{tabular}{|c|c|c|c|c|}
\hline EIXO & COMPETÊNCIAS & COMPONENTES & MÉTODOS DE APRENDIZADO & $\begin{array}{l}\text { MÉTODOS DE } \\
\text { AVALIAÇÃOO }\end{array}$ \\
\hline \multirow{5}{*}{$\begin{array}{l}\text { ABORDAGEM } \\
\text { INDIVIDUAL }\end{array}$} & $\begin{array}{l}\text { Adotar abordagem } \\
\text { centrada na pessoa }\end{array}$ & $\begin{array}{l}\text { Definir a agenda do paciente e a } \\
\text { experiência com o adoecer. } \\
\text { Identificar e esclarecer as metas e } \\
\text { prioridades do paciente. } \\
\text { Negociar as prioridades do } \\
\text { paciente. } \\
\text { Desenvolver e monitorar os } \\
\text { contratos definidos com o paciente. } \\
\text { Equilibrar as necessidades dos } \\
\text { pacientes com os desfechos } \\
\text { desejáveis de tratamento. } \\
\text { Esclarecer o entendimento do } \\
\text { paciente e desenvolver um plano } \\
\text { conjunto de manejo. }\end{array}$ & $\begin{array}{l}\text { ^Estudo de texto: } \\
\text { Capítulo 13. Consulta e abordagem } \\
\text { centrada na pessoa. } \\
\text { Discussão de casos clínicos. } \\
\text { Exemplo do preceptor e do } \\
\text { professor especialista em MFC. } \\
\text { Dramatização. }\end{array}$ & $\begin{array}{l}\text { Teste de conhecimento } \\
\text { aplicado } \\
\text { Observação direta } \\
\text { Vídeogravação }\end{array}$ \\
\hline & $\begin{array}{l}\text { Realizar anamnese de } \\
\text { forma apropriada para o } \\
\text { internato. }\end{array}$ & $\begin{array}{l}\text { Demonstrar habilidade para coletar } \\
\text { informações sobre os principais } \\
\text { sintomas apresentados pelo } \\
\text { paciente. } \\
\text { Direcionar o foco da história } \\
\text { levando em conta o sintoma } \\
\text { principal. } \\
\text { Esclarecer sintomas pertinentes } \\
\text { associados, sinais de alerta e } \\
\text { fatores de risco. } \\
\text { Demonstrar através da natureza } \\
\text { e sequência das perguntas que } \\
\text { as hipóteses diagnósticas estão } \\
\text { sendo geradas e testadas. }\end{array}$ & $\begin{array}{l}\text { Discussão de casos clínicos. } \\
\text { Exemplo do preceptor e do } \\
\text { professor especialista em MFC. } \\
\text { Dramatização. } \\
\text { Relatório SOAP }\end{array}$ & $\begin{array}{l}\text { Teste de conhecimento } \\
\text { aplicado } \\
\text { Observação direta } \\
\text { Vídeogravação + Rela- } \\
\text { tório SOAP }\end{array}$ \\
\hline & $\begin{array}{l}\text { Elaborar lista de } \\
\text { diagnóstico diferencial } \\
\text { condizente com as } \\
\text { informações coletadas na } \\
\text { anamnese e exame físico. }\end{array}$ & $\begin{array}{l}\text { Realizar registro da lista de } \\
\text { diagnóstico diferencial condizente } \\
\text { com as informações coletadas na } \\
\text { anamneses e exame físico. }\end{array}$ & $\begin{array}{l}\text { *Estudo de texto: } \\
\text { Capítulo } 21 \text {. Medicina baseada em } \\
\text { evidências aplicada à prática do } \\
\text { MFC. } \\
\text { Discussão de casos clínicos. } \\
\text { Exemplo do preceptor e do } \\
\text { professor. } \\
\text { Dramatização. }\end{array}$ & $\begin{array}{l}\text { Teste de conhecimento } \\
\text { aplicado } \\
\text { Observação direta } \\
\text { Vídeogravação + Rela- } \\
\text { tório SOAP }\end{array}$ \\
\hline & $\begin{array}{l}\text { Reconhecer a } \\
\text { apresentação típica e } \\
\text { atípica das doenças } \\
\text { prevalentes na APS e das } \\
\text { doenças com risco de } \\
\text { morte. }\end{array}$ & & $\begin{array}{l}\text { *Estudo de texto: } \\
\text { Capitulo 3. A organização de Servi- } \\
\text { ços de Atenção Primária à Saúde. } \\
\text { Duncan }\end{array}$ & $\begin{array}{l}\text { Teste de conhecimento } \\
\text { aplicado } \\
\text { Observação direta } \\
\text { Vídeogravação + Rela- } \\
\text { tório SOAP }\end{array}$ \\
\hline & $\begin{array}{l}\text { Demonstrar uma } \\
\text { abordagem eficaz para a } \\
\text { apresentação de sintomas } \\
\text { sem explicação médica. }\end{array}$ & $\begin{array}{l}\text { Considera os efeitos colaterais dos } \\
\text { medicamentos e interações como } \\
\text { um contribuinte potencial para os } \\
\text { sintomas dos pacientes. }\end{array}$ & $\begin{array}{l}\text { *Estudo de texto: } \\
\text { Capítulo 24. Prevenção quaterná- } \\
\text { ria: primeiro não causar dano. } \\
\text { Capítulo 93. Prescrevendo na APS. } \\
\text { Capítulo 94. Como desprescrever } \\
\text { medicamentos. Discussão de } \\
\text { casos clínicos. } \\
\text { Exemplo do preceptor e do } \\
\text { professor especialista em MFC. } \\
\text { Dramatização. }\end{array}$ & $\begin{array}{l}\text { Teste de conhecimento } \\
\text { aplicado } \\
\text { Observação direta } \\
\text { Vídeogravação + Rela- } \\
\text { tório SOAP }\end{array}$ \\
\hline
\end{tabular}


Continuação Quadro 3.

\begin{tabular}{|c|c|c|c|c|}
\hline EIXO & COMPETÊNCIAS & COMPONENTES & MÉTODOS DE APRENDIZADO & $\begin{array}{l}\text { MÉTODOS DE } \\
\text { AVALIAÇÃ̃O }\end{array}$ \\
\hline \multirow{10}{*}{$\begin{array}{l}\text { ABORDAGEM } \\
\text { INDIVIDUAL }\end{array}$} & \multirow{9}{*}{$\begin{array}{l}\text { Demonstrar uma } \\
\text { abordagem eficaz para a } \\
\text { apresentação de sintomas } \\
\text { sem explicação médica. }\end{array}$} & $\begin{array}{l}\text { Considera sintomas sem } \\
\text { explicação médica no diagnóstico } \\
\text { diferencial. }\end{array}$ & $\begin{array}{l}\text { *Estudo de texto: } \\
\text { Capítulo 218. Somatização e } \\
\text { sintomas sem explicação médica. } \\
\text { Discussão de casos clínicos. } \\
\text { Exemplo do preceptor e do } \\
\text { professor especialista em MFC. } \\
\text { Dramatização. }\end{array}$ & $\begin{array}{l}\text { Teste de conhecimento } \\
\text { aplicado } \\
\text { Observação direta } \\
\text { Vídeogravação + Rela- } \\
\text { tório SOAP }\end{array}$ \\
\hline & & $\begin{array}{l}\text { Explora ativamente o sintoma } \\
\text { indiferenciado como uma possível } \\
\text { complicação do diagnóstico } \\
\text { estabelecido. }\end{array}$ & $\begin{array}{l}\text { *Estudo de texto: } \\
\text { Capítulo } 218 \text {. Somatização e } \\
\text { sintomas sem explicação médica. } \\
\text { Discussão de casos clínicos. } \\
\text { Exemplo do preceptor e do } \\
\text { professor especialista em MFC. } \\
\text { Dramatização. }\end{array}$ & $\begin{array}{l}\text { Teste de conhecimento } \\
\text { aplicado } \\
\text { Observação direta } \\
\text { Vídeogravação + Rela- } \\
\text { tório SOAP }\end{array}$ \\
\hline & & $\begin{array}{l}\text { Avalia as possíveis contribuições } \\
\text { de ambos os fatores orgânicos e } \\
\text { psicossociais no desenvolvimento } \\
\text { de sintomas sem explicação } \\
\text { médica. }\end{array}$ & $\begin{array}{l}\text { Discussão de casos clínicos. } \\
\text { Exemplo do preceptor e do } \\
\text { professor especialista em MFC. } \\
\text { Dramatização. }\end{array}$ & $\begin{array}{l}\text { Teste de conhecimento } \\
\text { aplicado } \\
\text { Observação direta } \\
\text { Vídeogravação + Rela- } \\
\text { tório SOAP }\end{array}$ \\
\hline & & $\begin{array}{l}\text { Explora contexto cultural e social } \\
\text { do paciente para entender como } \\
\text { estes podem influenciar a apresen- } \\
\text { tação do (s) seu (s) sintoma (s). }\end{array}$ & $\begin{array}{l}\text { Discussão de casos clínicos. } \\
\text { Exemplo do preceptor e do } \\
\text { professor especialista em MFC. } \\
\text { Dramatização. }\end{array}$ & $\begin{array}{l}\text { Observação direta } \\
\text { Vídeogravação + Rela- } \\
\text { tório SOAP }\end{array}$ \\
\hline & & $\begin{array}{l}\text { Mostra sensibilidade cultural e } \\
\text { consciência do contexto social do } \\
\text { paciente na implementação de um } \\
\text { plano de tratamento. }\end{array}$ & $\begin{array}{l}\text { Discussão de casos clínicos. } \\
\text { Exemplo do preceptor. }\end{array}$ & $\begin{array}{l}\text { Observação direta } \\
\text { Vídeogravação + Rela- } \\
\text { tório SOAP }\end{array}$ \\
\hline & & $\begin{array}{l}\text { Emprega uma estratégia de } \\
\text { assistência ao paciente ao } \\
\text { longo do tempo para facilitar } \\
\text { o diagnóstico e tratamento de } \\
\text { doenças que apresentam em um } \\
\text { estágio indiferenciado. }\end{array}$ & $\begin{array}{l}\text { Discussão de casos clínicos. } \\
\text { Exemplo do preceptor. }\end{array}$ & $\begin{array}{l}\text { Observação direta } \\
\text { Vídeogravação + Rela- } \\
\text { tório SOAP }\end{array}$ \\
\hline & & $\begin{array}{l}\text { Compromete-se apenas a } \\
\text { investigações diagnósticas que } \\
\text { são justificáveis em termos de } \\
\text { probabilidade pré-teste de doença, } \\
\text { melhor evidência disponível e } \\
\text { custo. }\end{array}$ & $\begin{array}{l}\text { *Estudo de texto: } \\
\text { Capítulo 21. Medicina baseada em } \\
\text { evidências aplicada à prática do } \\
\text { MFC. } \\
\text { Discussão de casos clínicos. } \\
\text { Exemplo do preceptor e do } \\
\text { professor especialista em MFC. } \\
\text { Dramatização. }\end{array}$ & $\begin{array}{l}\text { Teste de conhecimento } \\
\text { aplicado } \\
\text { Observação direta } \\
\text { Vídeogravação + Rela- } \\
\text { tório SOAP }\end{array}$ \\
\hline & & $\begin{array}{l}\text { Verifica com os pacientes para } \\
\text { garantir se entendeu o sintoma } \\
\text { sem explicação médica. }\end{array}$ & $\begin{array}{l}\text { Discussão de casos clínicos. } \\
\text { Exemplo do preceptor e do } \\
\text { professor especialista em MFC. } \\
\text { Dramatização. }\end{array}$ & $\begin{array}{l}\text { Observação direta } \\
\text { Vídeogravação }\end{array}$ \\
\hline & & $\begin{array}{l}\text { Planeja um acompanhamento } \\
\text { adequado dos pacientes com } \\
\text { sintomas sem explicação médica. }\end{array}$ & $\begin{array}{l}\text { Discussão de casos clínicos. } \\
\text { Exemplo do preceptor e do } \\
\text { professor especialista em MFC. } \\
\text { Dramatização. }\end{array}$ & $\begin{array}{l}\text { Observação direta } \\
\text { Vídeogravação + Rela- } \\
\text { tório SOAP }\end{array}$ \\
\hline & $\begin{array}{l}\text { Demonstrar uma aborda- } \\
\text { gem eficaz para a apresen- } \\
\text { tação de doença aguda } \\
\text { autolimitada e doença } \\
\text { potencialmente fatal. }\end{array}$ & $\begin{array}{l}\text { Formular um diagnóstico diferen- } \\
\text { cial que inclua o diagnóstico mais } \\
\text { provável (tendo em conta a preva- } \\
\text { lência da doença na população), } \\
\text { uma hierarquia de alternativas } \\
\text { possíveis, e a possibilidade mais } \\
\text { grave ou com risco de vida. }\end{array}$ & $\begin{array}{l}\text { *Estudo de texto: } \\
\text { Seção XXIX. Problemas com Risco } \\
\text { de Morte. } \\
\text { Discussão de casos clínicos. } \\
\text { Exemplo do preceptor e do } \\
\text { professor especialista em MFC. } \\
\text { Dramatização. }\end{array}$ & $\begin{array}{l}\text { Teste de conhecimento } \\
\text { aplicado } \\
\text { Observação direta } \\
\text { Vídeogravação + Rela- } \\
\text { tório SOAP }\end{array}$ \\
\hline
\end{tabular}


Continuação Quadro 3.

\begin{tabular}{|c|c|c|c|c|}
\hline EIXO & COMPETÊNCIAS & COMPONENTES & MÉTODOS DE APRENDIZADO & $\begin{array}{l}\text { MÉTODOS DE } \\
\text { AVALIAÇÃO }\end{array}$ \\
\hline \multirow{9}{*}{$\begin{array}{l}\text { ABORDAGEM } \\
\text { INDIVIDUAL }\end{array}$} & \multirow{9}{*}{$\begin{array}{l}\text { Demonstrar uma aborda- } \\
\text { gem eficaz para a apresen- } \\
\text { tação de doença aguda } \\
\text { autolimitada e doença } \\
\text { potencialmente fatal. }\end{array}$} & $\begin{array}{l}\text { Demonstrar uma compreensão da } \\
\text { história natural e prognóstico do } \\
\text { diagnóstico estabelecido quando } \\
\text { tratados e não tratados. }\end{array}$ & $\begin{array}{l}\text { *Estudo de texto: } \\
\text { Seção XXIX. Problemas com Risco } \\
\text { de Morte. } \\
\text { Discussão de casos clínicos. } \\
\text { Exemplo do preceptor e do } \\
\text { professor especialista em MFC. } \\
\text { Dramatização. }\end{array}$ & $\begin{array}{l}\text { Teste de conhecimento } \\
\text { aplicado } \\
\text { Observação direta } \\
\text { Vídeogravação + Rela- } \\
\text { tório SOAP }\end{array}$ \\
\hline & & $\begin{array}{l}\text { Localizar as provas pertinentes, } \\
\text { quando necessário para o } \\
\text { diagnóstico e tratamento. }\end{array}$ & $\begin{array}{l}\text { *Estudo de texto: } \\
\text { Seção XXIX. Problemas com Risco } \\
\text { de Morte. } \\
\text { Capítulo } 21 . \text { Medicina baseada em } \\
\text { evidências aplicada à prática do } \\
\text { MFC. } \\
\text { Discussão de casos clínicos. } \\
\text { Exemplo do preceptor e do } \\
\text { professor especialista em MFC. } \\
\text { Dramatização. }\end{array}$ & $\begin{array}{l}\text { Teste de conhecimento } \\
\text { aplicado } \\
\text { Observação direta } \\
\text { Vídeogravação + Rela- } \\
\text { tório SOAP }\end{array}$ \\
\hline & & $\begin{array}{l}\text { Discutir a evidência mais relevante } \\
\text { para a terapia médica para o } \\
\text { diagnóstico. }\end{array}$ & $\begin{array}{l}\text { *Estudo de texto: } \\
\text { Seção XXIX. Problemas com Risco } \\
\text { de Morte. } \\
\text { Capítulo } 21 . \text { Medicina baseada em } \\
\text { evidências aplicada à prática do } \\
\text { MFC. } \\
\text { Discussão de casos clínicos. } \\
\text { Exemplo do preceptor e do } \\
\text { professor especialista em MFC. } \\
\text { Dramatização. }\end{array}$ & $\begin{array}{l}\text { Teste de conhecimento } \\
\text { aplicado } \\
\text { Observação direta } \\
\text { Vídeogravação + Rela- } \\
\text { tório SOAP }\end{array}$ \\
\hline & & $\begin{array}{l}\text { Recomendar somente produtos } \\
\text { farmacêuticos que são claramente } \\
\text { indicados para o diagnóstico. }\end{array}$ & $\begin{array}{l}\text { *Estudo de texto: } \\
\text { Seção XXIX. Problemas com Risco } \\
\text { de Morte. } \\
\text { Capítulo } 21 . \text { Medicina baseada em } \\
\text { evidências aplicada à prática do } \\
\text { MFC. } \\
\text { Discussão de casos clínicos. } \\
\text { Exemplo do preceptor e do } \\
\text { professor especialista em MFC. } \\
\text { Dramatização. }\end{array}$ & $\begin{array}{l}\text { Teste de conhecimento } \\
\text { aplicado } \\
\text { Observação direta } \\
\text { Vídeogravação + Rela- } \\
\text { tório SOAP }\end{array}$ \\
\hline & & $\begin{array}{l}\text { Reforçar ao paciente a importância } \\
\text { da terapia não farmacológica } \\
\text { adequada. }\end{array}$ & $\begin{array}{l}\text { Discussão de casos clínicos. } \\
\text { Exemplo do preceptor e do } \\
\text { professor especialista em MFC. }\end{array}$ & $\begin{array}{l}\text { Observação direta } \\
\text { Vídeogravação }\end{array}$ \\
\hline & & $\begin{array}{l}\text { Explicar ao paciente quando e/ou } \\
\text { porque eles precisam de acompa- } \\
\text { nhamento clínico. }\end{array}$ & $\begin{array}{l}\text { Discussão de casos clínicos. } \\
\text { Exemplo do preceptor e do } \\
\text { professor especialista em MFC. }\end{array}$ & $\begin{array}{l}\text { Observação direta } \\
\text { Vídeogravação }\end{array}$ \\
\hline & & $\begin{array}{l}\text { Gerenciar a maioria dos diagnósti- } \\
\text { cos frequentes, sem a necessidade } \\
\text { de encaminhamento. }\end{array}$ & $\begin{array}{l}\text { Discussão de casos clínicos. } \\
\text { Exemplo do preceptor e do } \\
\text { professor especialista em MFC. }\end{array}$ & $\begin{array}{l}\text { Observação direta } \\
\text { Vídeogravação }\end{array}$ \\
\hline & & $\begin{array}{l}\text { Listar e demonstrar o uso das habi- } \\
\text { lidades essenciais, equipamentos } \\
\text { e medicamentos necessários para } \\
\text { lidar com a vida em risco, condi- } \\
\text { ções agudas na APS. }\end{array}$ & $\begin{array}{l}\text { Discussão de casos clínicos. } \\
\text { Exemplo do preceptor e do } \\
\text { professor especialista em MFC. }\end{array}$ & $\begin{array}{l}\text { Observação direta } \\
\text { Vídeogravação }\end{array}$ \\
\hline & & $\begin{array}{l}\text { Providenciar acesso seguro e } \\
\text { imediato serviços de emergência } \\
\text { para o paciente com uma condição } \\
\text { potencialmente fatal. }\end{array}$ & $\begin{array}{l}\text { *Estudo de texto: } \\
\text { Capitulo 229. Princípios da prática } \\
\text { da medicina pré-hospitalar. } \\
\text { Discussão de casos clínicos. } \\
\text { Exemplo do preceptor e do } \\
\text { professor especialista em MFC. }\end{array}$ & $\begin{array}{l}\text { Teste de conhecimento } \\
\text { aplicado. } \\
\text { Observação direta } \\
\text { Vídeogravação }\end{array}$ \\
\hline
\end{tabular}


Continuação Quadro 3.

\begin{tabular}{|c|c|c|c|c|}
\hline EIXO & COMPETÊNCIAS & COMPONENTES & MÉTODOS DE APRENDIZADO & $\begin{array}{l}\text { MÉTODOS DE } \\
\text { AVALIAÇÃO }\end{array}$ \\
\hline \multirow{11}{*}{$\begin{array}{l}\text { ABORDAGEM } \\
\text { INDIVIDUAL }\end{array}$} & $\begin{array}{l}\text { Demonstrar uma aborda- } \\
\text { gem eficaz para a apresen- } \\
\text { tação de doença aguda } \\
\text { autolimitada e doença } \\
\text { potencialmente fatal. }\end{array}$ & $\begin{array}{l}\text { Compartilhar informações-chave } \\
\text { com os serviços de emergência } \\
\text { quando se refere a pacientes com } \\
\text { condições de risco de vida. }\end{array}$ & $\begin{array}{l}\text { Discussão de casos clínicos. } \\
\text { Exemplo do preceptor e do } \\
\text { professor especialista em MFC. }\end{array}$ & $\begin{array}{l}\text { Observação direta } \\
\text { Vídeogravação }\end{array}$ \\
\hline & \multirow{10}{*}{$\begin{array}{l}\text { Demonstrar uma aborda- } \\
\text { gem eficaz em relação às } \\
\text { doenças crônicas. }\end{array}$} & $\begin{array}{l}\text { Rastrear apropriadamente } \\
\text { pacientes assintomáticos, de } \\
\text { acordo com as recomendações } \\
\text { baseadas em evidências mais } \\
\text { relevantes. }\end{array}$ & $\begin{array}{l}\text { *Estudo de texto: } \\
\text { Capitulo } 56 \text {. Estratégias preven- } \\
\text { tivas para doenças crônicas não } \\
\text { transmissíveis. } \\
\text { Capítulo } 60 \text {. Rastreamento de } \\
\text { doença. } \\
\text { Discussão de casos clínicos. } \\
\text { Exemplo do preceptor e do } \\
\text { professor especialista em MFC. }\end{array}$ & $\begin{array}{l}\text { Teste de conhecimento } \\
\text { aplicado } \\
\text { Observação direta } \\
\text { Vídeogravação + Rela- } \\
\text { tório SOAP }\end{array}$ \\
\hline & & $\begin{array}{l}\text { Interpretar corretamente os } \\
\text { resultados dos testes utilizados } \\
\text { para diagnosticar doenças } \\
\text { crônicas. }\end{array}$ & $\begin{array}{l}\text { Discussão de casos clínicos. } \\
\text { Exemplo do preceptor e do } \\
\text { professor especialista em MFC. }\end{array}$ & $\begin{array}{l}\text { Teste de conhecimento } \\
\text { aplicado } \\
\text { Observação direta } \\
\text { Vídeogravação + Rela- } \\
\text { tório SOAP }\end{array}$ \\
\hline & & $\begin{array}{l}\text { Certificar-se que o paciente } \\
\text { preencha os critérios diagnósticos } \\
\text { para a doença crônica antes de } \\
\text { confirmar o diagnóstico. }\end{array}$ & $\begin{array}{l}\text { Discussão de casos clínicos. } \\
\text { Exemplo do preceptor e do } \\
\text { professor especialista em MFC. }\end{array}$ & $\begin{array}{l}\text { Teste de conhecimento } \\
\text { aplicado } \\
\text { Observação direta } \\
\text { Vídeogravação + Rela- } \\
\text { tório SOAP }\end{array}$ \\
\hline & & $\begin{array}{l}\text { Informar corretamente os objetivos } \\
\text { do tratamento das doenças } \\
\text { crônicas comuns, de acordo com } \\
\text { os consensos mais relevantes. }\end{array}$ & $\begin{array}{l}\text { Discussão de casos clínicos. } \\
\text { Exemplo do preceptor e do } \\
\text { professor especialista em MFC. }\end{array}$ & $\begin{array}{l}\text { Observação direta } \\
\text { Vídeogravação }\end{array}$ \\
\hline & & $\begin{array}{l}\text { Reconhecer as principais com- } \\
\text { plicações das doenças crônicas } \\
\text { comuns e como monitorá-las ade- } \\
\text { quadamente. }\end{array}$ & $\begin{array}{l}\text { Discussão de casos clínicos. } \\
\text { Exemplo do preceptor e do } \\
\text { professor especialista em MFC. }\end{array}$ & $\begin{array}{l}\text { Teste de conhecimento } \\
\text { aplicado } \\
\text { Observação direta } \\
\text { Vídeogravação + Rela- } \\
\text { tório SOAP }\end{array}$ \\
\hline & & $\begin{array}{l}\text { Considerar a possibilidade de uma } \\
\text { doença crônica estabelecida poder } \\
\text { contribuir para um novo sintoma. }\end{array}$ & $\begin{array}{l}\text { Discussão de casos clínicos. } \\
\text { Exemplo do preceptor e do } \\
\text { professor especialista em MFC. }\end{array}$ & $\begin{array}{l}\text { Teste de conhecimento } \\
\text { aplicado } \\
\text { Observação direta } \\
\text { Vídeogravação + Rela- } \\
\text { tório SOAP }\end{array}$ \\
\hline & & $\begin{array}{l}\text { Recomendar, quando indicado, } \\
\text { farmacoterapia para alívio de } \\
\text { sintomas, para alcançar objetivos } \\
\text { do tratamento ou prevenir } \\
\text { complicações. }\end{array}$ & $\begin{array}{l}\text { Discussão de casos clínicos. } \\
\text { Exemplo do preceptor e do } \\
\text { professor especialista em MFC. }\end{array}$ & $\begin{array}{l}\text { Teste de conhecimento } \\
\text { aplicado } \\
\text { Observação direta } \\
\text { Vídeogravação + Rela- } \\
\text { tório SOAP }\end{array}$ \\
\hline & & $\begin{array}{l}\text { Abordar a doença crônica do } \\
\text { paciente, mesmo quando a queixa } \\
\text { da consulta não parece estar } \\
\text { associada à condição. }\end{array}$ & $\begin{array}{l}\text { Discussão de casos clínicos. } \\
\text { Exemplo do preceptor e do } \\
\text { professor especialista em MFC. }\end{array}$ & Observação direta \\
\hline & & $\begin{array}{l}\text { Propor que o paciente estabeleça } \\
\text { objetivos alcançáveis de mudança } \\
\text { de estilo de vida, no sentido de } \\
\text { maximizar sua capacidade de } \\
\text { controle da doença. }\end{array}$ & $\begin{array}{l}\text { Discussão de casos clínicos. } \\
\text { Exemplo do preceptor e do } \\
\text { professor especialista em MFC. }\end{array}$ & $\begin{array}{l}\text { Teste de conhecimento } \\
\text { aplicado } \\
\text { Observação direta } \\
\text { Vídeogravação + Rela- } \\
\text { tório SOAP }\end{array}$ \\
\hline & & $\begin{array}{l}\text { Enfatizar a educação do paciente } \\
\text { a respeito da sua doença crônica } \\
\text { usando ferramentas e recursos } \\
\text { disponíveis e os empoderar quanto } \\
\text { à sua doença. }\end{array}$ & $\begin{array}{l}\text { Discussão de casos clínicos. } \\
\text { Exemplo do preceptor e do } \\
\text { professor especialista em MFC. }\end{array}$ & Observação direta \\
\hline
\end{tabular}


Continuação Quadro 3.

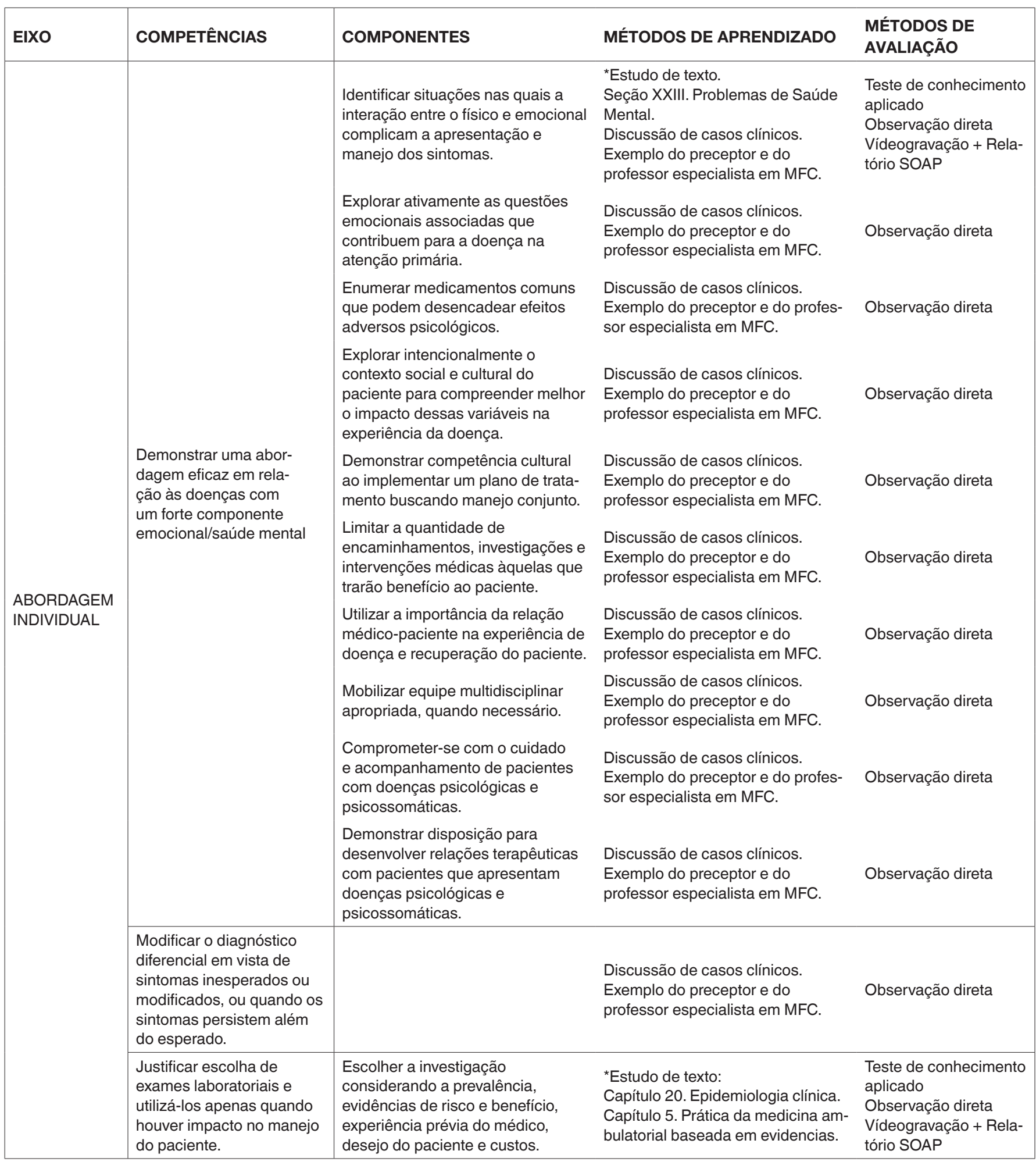


Continuação Quadro 3.

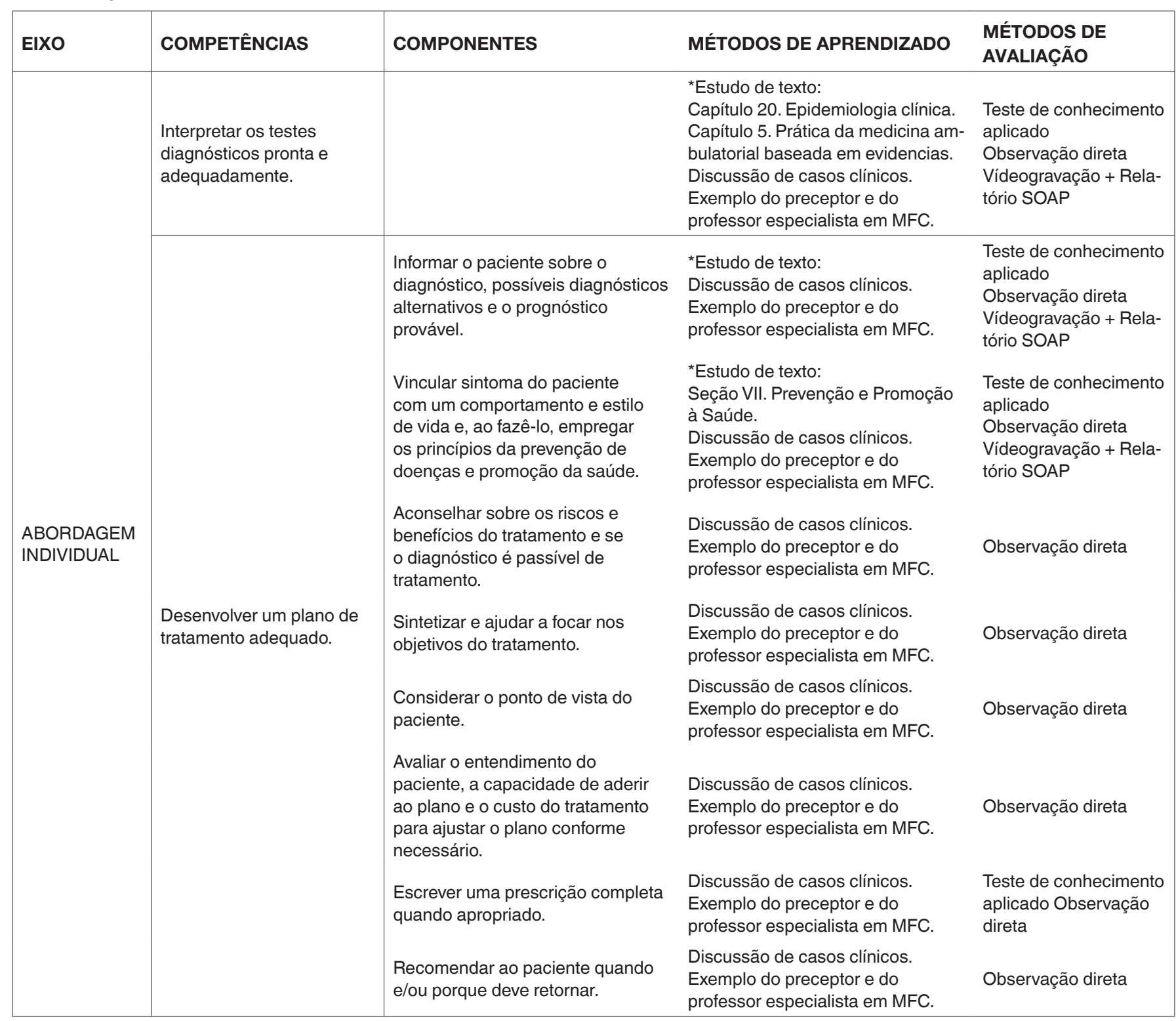

A ideia de eixo remete ao conceito de dar uma direção a uma competência, possibilitando a avaliação da aquisição dos conhecimentos, habilidades e atitudes ao longo do tempo de forma independente. Apesar de poderem ser avaliados independentemente, o desempenho ótimo em apenas um eixo caracteriza um conjunto de competências não satisfatório. Por outro lado, os eixos se relacionam entre si de forma a potencializar o desenvolvimento das competências mutuamente. Como exemplo, um estudante que desenvolve a competência de realizar anamnese de forma apropriada para o internato no eixo de Abordagem Individual também acaba demonstrando habilidades de comunicação efetiva, profissional e sem preconceitos contemplada no eixo Geral. 
Quadro 4. Continuação da Matriz de Competências do Internato em Medicina de Família e Comunidade no Eixo Abordagem Familiar.

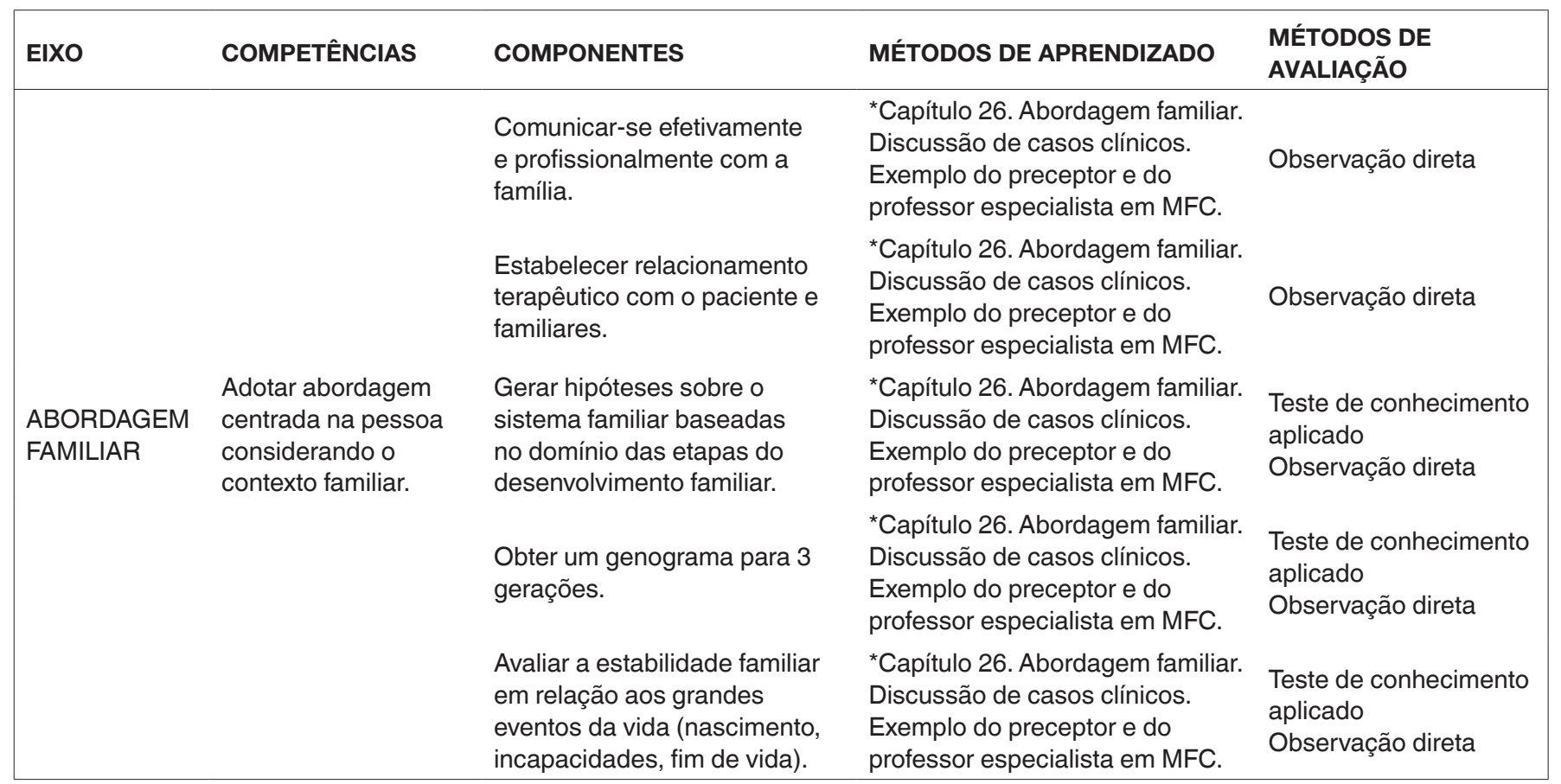

Quadro 5. Continuação da Matriz de Competências do Internato em Medicina de Família e Comunidade no Eixo Abordagem Comunitária.

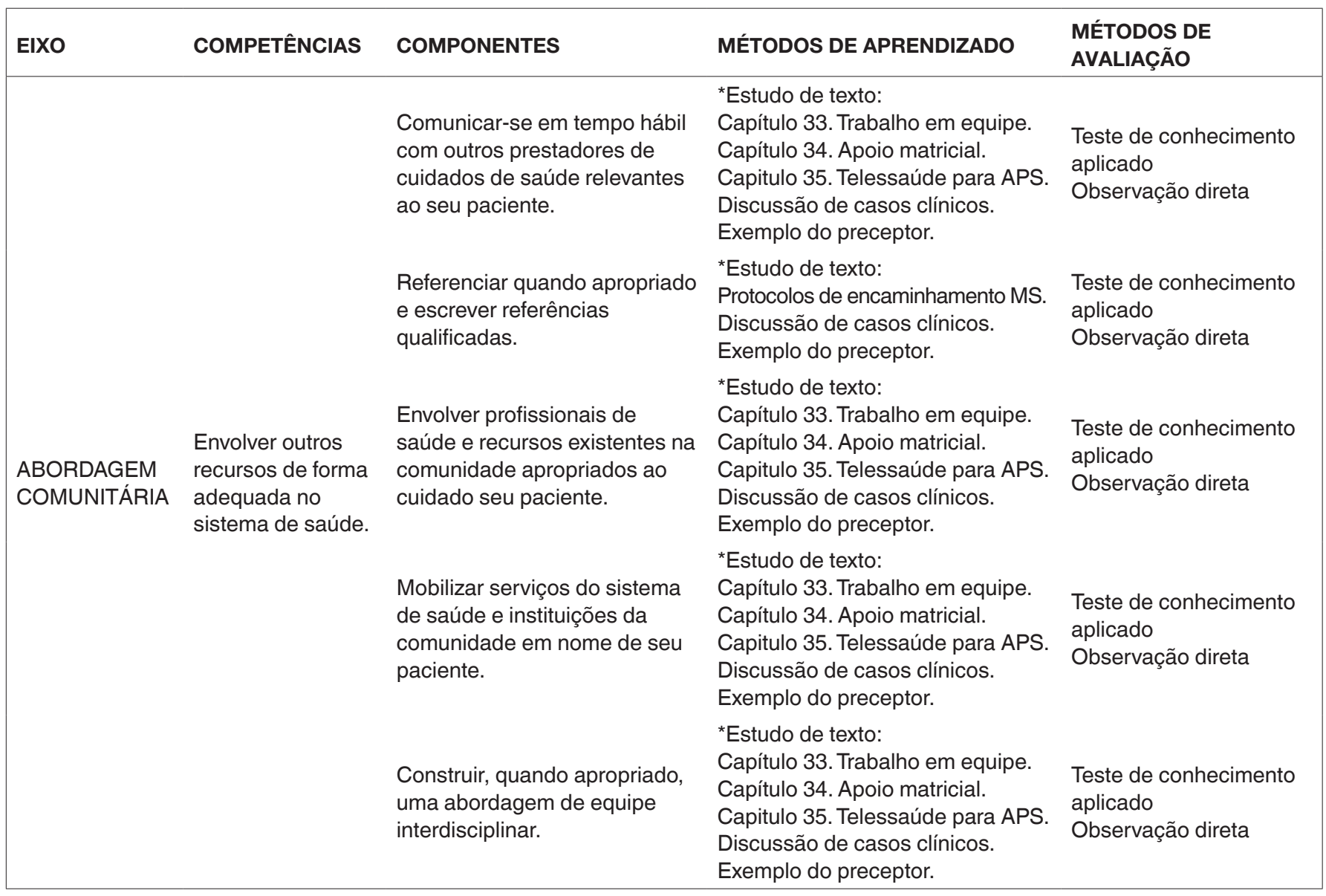


O eixo Geral compreende competências que são referenciais filosóficos e pilares da especialidade. Já o eixo de Abordagem Individual, traduz a aplicação dos referenciais técnicos para prática clínica com enfoque na pessoa, em contrapartida ao enfoque apenas na doença. Por fim, tanto no eixo de Abordagem Familiar como no de Abordagem Comunitária, o estudante deve considerar um contexto mais amplo do atendimento, como a influência da família, assim como o possível envolvimento de outros profissionais no cuidado da pessoa.

Os métodos de ensino utilizados para o desenvolvimento das competências e os métodos de avaliação em cada eixo estão apresentados nos Quadros 2 a 5. A avaliação final do estágio ainda é somativa. Entretanto, é realizada avaliação formativa aliada à somativa na apresentação da videogravação das consultas, na postagem dos relatórios, nos testes de conhecimento aplicado e na observação direta pelo preceptor nas unidades de estágio.

\section{DISCUSSÃO}

Esse artigo apresenta a experiência dos professores do internato em MFC da UFCSPA em elaborar uma matriz de competências de acordo com sua realidade de ensino em uma Instituição de Ensino Superior com currículo tradicional.

Entre os componentes que mais se destacam na Matriz está o desenvolvimento da consciência de ser um eterno aprendiz frente aos problemas enfrentados na atenção primária. A atenção primária é a porta de entrada prioritária no SUS. A atuação nesse nível do sistema de saúde demanda abordagem de problemas complexos e diversos os quais exigem do médico habilidade e atitude em lidar com situações inesperadas buscando soluções baseadas nas necessidades das pessoas. Assim, desenvolver a capacidade de identificar e conciliar a sua agenda profissional com a agenda do paciente, compartilhar a decisão em relação ao plano terapêutico, estar atento ao perfil da comunidade, manter-se atualizado e conhecer sua rede de atenção, são aspectos fundamentais para garantir a eficiência do atendimento médico.

Fica evidente na Matriz que praticamente todas as competências envolvem habilidades e atitudes comunicacionais. Isso deixa claro aos alunos, professores e preceptores a necessidade de desenvolver habilidades em se comunicar com as pessoas, seus familiares, com a comunidade, equipamentos sociais, equipe de trabalho local e equipes em outros níveis do sistema. Os alunos e professores, geralmente, valorizam aspectos relacionados ao conhecimento teórico especializado do atendimento. Colocar na agenda curricular competências que abrangem a capacidade de se relacionar com pessoas possibilita a conscientização da importância de desenvolvê-las. Uma vez que o estudante entende as competências esperadas, ele também terá autonomia para buscá-las.

Como limitação, está a construção dessa Matriz somente pelo núcleo de professores do internato, sem a participação direta e ativa dos preceptores e internos. Por outro lado, alguns dos professores são preceptores, participaram do processo de elaboração do currículo baseado em competências da SBMFC e contribuíram com sua expertise em preceptoria para tradução das recomendações à realidade do internato em nossa Instituição.

Além disso, houve uma contribuição indireta dos preceptores e estudantes com as respostas a questionário semiestruturado de avaliação do programa. Com essa avaliação, ficou evidente que há discrepâncias entre as experiências de ensino-aprendizagem dos internos nas unidades básicas de saúde, 
desconhecimento dos objetivos específicos do estágio e críticas em relação ao processo de avaliação. Conhecer o estado atual do currículo foi o motivador fundamental para a elaboração dessa Matriz, a qual explicitou os métodos de ensino e de avaliação atualmente utilizados, o que possibilitará a revisão da validade de tais métodos.

O currículo baseado em competências vem sendo utilizado pelas escolas médicas na Europa e América do Norte. Essas Escolas utilizam recomendações de Instituições internacionais destinadas a apoiar os programas a implementar a avaliação baseada em competências. ${ }^{9} \mathrm{O}$ desenvolvimento da Matriz de competências do internato em nossa instituição baseou-se também nesses critérios internacionais fazendo as devidas adaptações para o contexto brasileiro.

Um estudo realizado pela European Academy of Teachers of General Practice and Family Medicine (EURACT) ${ }^{26}$ definiu 15 competências básicas a serem desenvolvidas no internato em MFC em cenários nos quais os estágios sejam oferecidos em períodos inferiores a quatro meses. Nossa Matriz está de acordo e se assemelha à definida por esse estudo.

Na América Latina ${ }^{11,12,21,22}$ e no Brasil ${ }^{13,27}$ as discussões sobre o currículo baseado em competências iniciaram-se na década de 90. Desde então, algumas escolas implementaram o currículo por competências como a Universidade Estadual de Londrina (UEL), ${ }^{14}$ Universidade da Cidade de São Paulo (UNICID) ${ }^{9}$ e Faculdade de Medicina de Marília (FAMEMA). ${ }^{15}$ A matriz de competências do internato em MFC nessas escolas não está restrita a esse momento no curso. Ela faz parte de um processo de desenvolvimento de conhecimentos, habilidades e atitudes ao longo de todos os anos utilizando metodologias ativas de ensino aprendizagem, como abordagem baseada em problemas e problematização.

A experiência na elaboração do currículo baseado em competências nessas escolas difere da experiência relatada nesse artigo, pois estão baseadas num projeto pedagógico consistente com o processo de aquisição de competências profissionais de forma longitudinal e interdisciplinar ao longo do curso.

A adaptação das recomendações internacionais e nacionais referentes à residência em MFC ao contexto do internato visou definir competências básicas em MFC a serem desenvolvidas pelos estudantes enquanto graduandos de modo a possibilitar aperfeiçoamento dessas competências na residência médica. O período de três meses de internato em nossa Instituição, certamente, é insuficiente para desenvolvimento pleno das competências definidas. Espera-se que a elaboração da dessa Matriz contribua para a discussão sobre a reorganização curricular baseada em competências em nossa Instituição e aponte para a necessidade da integração longitudinal ao longo do currículo do curso, com outros cursos e com a residência médica.

\section{Conclusão}

Com a definição da Matriz, as competências, antes desenvolvidas de forma intuitiva, foram organizadas de forma a permitir a avaliação da validade dos métodos de ensino-aprendizagem e dos instrumentos de avaliação. Assim, espera-se otimizar o processo de incorporação do ensino baseado em competências no currículo em nossa Instituição. Cientes de que essa Matriz estará em contínua construção em nosso contexto, espera-se que possa ser utilizada e aprimorada por outras escolas médicas em cenários semelhantes. A Matriz de competências elaborada é pública, sendo a avaliação externa por pares essencial para sua validação e confiabilidade. 


\section{Referências}

1. Gruppen LD, Mangrulkar RS, Kolars JC. The promise of competency-based education in the health professions for improving global health. Hum Resour Health. 2012;10:43. DOI: http://dx.doi.org/10.1186/1478-4491-10-43

2. Santos WS. Organização curricular baseada em competência na educação médica. Rev Bras Educ Médica.2011;35(1):8692. DOI: http://dx.doi.org/10.1590/S0100-55022011000100012

3. Carraccio C, Wolfsthal SD, Englander R, Ferentz K, Martin C. Shifting paradigms: from Flexner to competencies. Acad Med. 2002;77(5):361-7. DOI: http://dx.doi.org/10.1097/00001888-200205000-00003

4. Quintero GA. Medical education and the healthcare system--why does the curriculum need to be reformed? BMC Med. 2014;12:213.

5. World Health Organization. The world health report 2008: primary health care now more than ever. [Internet]. Geneva:World Health Organization; 2008. [cited 2017 May 2]. Available from: http://www.who.int/whr/2008/whr08_en.pdf

6. Perrenoud P. Ensinar: agir na urgência, decidir na incerteza: saberes e competências em uma profissão. 2a ed. Porto Alegre: Artmed; 2001.

7. Scheffer M, Biancarelli A, Cassenote A. Demografia médica no Brasil 2015 [Internet]. Departamento de Medicina Preventiva da Faculdade de Medicina da USP; Conselho Regional de Medicina do Estado de São Paulo; 2015 p. 284. Report No.: ISBN: 978-85-89656-22-1. [cited 2017 May 2]. Available at: http://www.usp.br/agen/wp-content/uploads/ DemografiaMedica30nov2015.pdf

8. Association for Medical Education in Europe (AMEE). Outcome-based education. No 14. Association for Medical Education in Europe. Dundee: AMEE; 1999.

9. Bollela VR, Machado JLM. Bridging the gaps [Internet]. Vol. 30. [cited 2016 Jun 26]. Available from: https://www.researchgate. net/profile/Valdes_Bollela/publication/259677053_Internato_Baseado_em_Competencias_Bridging_the_gaps_(2010)/ links/0deec52d50c12c96db000000.pdf

10. Albanese MA, Mejicano G, Anderson WM, Gruppen L. Building a competency-based curriculum: the agony and the ecstasy. Adv Health Sci Educ Theory Pract. 2010;15(3):439-54. DOI: http://dx.doi.org/10.1007/s10459-008-9118-2

11. Schmal SR, Ruiz-Tagle AA. Una metodología para el diseño de un currículo orientado a las competencias. Ingeniare Rev Chil Ing. 2008;16(1):147-58. DOI: http://dx.doi.org/10.4067/S0718-33052008000100004

12. Risco de Domínguez G. Design and implementation of a competency-based curriculum for medical education. Rev Peru Med Exp Salud Publica. 2014;31(3):572-81.

13. Braid LMC, Machado MFAS, Aranha AC. State of the art of curriculum research relating to healthcare professional training courses: a survey on articles published between 2005 and 2011. Interface (Botucatu). 2012;16(42):679-92. DOI: http:// dx.doi.org/10.1590/S1414-32832012000300008

14. Campos JJB. Novos currículos de Medicina na FAMEMA e na UEL: uma construção permanente rumo à realidade presente e futura [Internet]. 2001 [cited 2016 Jul 3]. Available from: http://www.uel.br/ccs/olhomagico/v8n2/Joao2.htm

15. Feuerwerker LCM. Mudanças na educação médica e residência médica no Brasil. São Paulo: Hucitec; 1998. 190 p.

16. An International Association For Medical Education - AMEE [Internet]. [cited 2016 Dec 28]. Available from: https://www. amee.org/home

17. Allen S, Anim T, Anthony E, Araujo D, Beebe D, Dostal J, et al. The Family Medicine Milestone Project. The Accreditation Council for Graduate Medical Education and The American Board of Family Medicine [Internet]. 2015 [cited 2016 Jul 3]. Available from: https://www.acgme.org/Portals/0/PDFs/Milestones/FamilyMedicineMilestones.pdf

18. Medical Education - Initiatives - AAMC [Internet]. [cited 2016 Dec 28]. Available from: https://www.aamc.org/initiatives/meded/

19. Chair DT, Konkin J, Parsons E, Saucier D, Shaw L, Walsh A, et al. Canadian Medical Education (CanMEDS)-FamilyMedicine [Internet]. 2009. [cited 2017 May 2]. Available from: http://www.cfpc.ca/uploadedFiles/Education/CanMeds\%20FM\%20 Eng.pdf

20. Core EPAs - Initiatives - AAMC [Internet]. [cited 2016 Dec 29]. Available from: https://www.aamc.org/initiatives/coreepas/

21. Zahlout AJM, Uzcátegui Z, Pérez-González JF. Exploración de un modelo de la competencia médica en alumnos de sexto año de la Escuela de Medicina "Luis Razetti". Rev Fac Med. 2006;29(2):184-93. 
22. Ribeiro ECO. A educação dos profissionais de saúde na América Latina: teoria e prática de um movimento de mudança. Interface (Botucatu). 2000;4(7):139-42. DOI: http://dx.doi.org/10.1590/S1414-32832000000200014

23. Ministério da Educação. Conselho Nacional de Educação. Resoluçãon.3, de 20 de junho de 2014. Institui Diretrizes Curriculares Nacionais do Curso de Graduação em Medicina e dá outras providências. [Internet]. Ministério da Educação;2014 [acesso 2016 Jun 25]. Disponível em: http://portal.mec.gov.br/index.php?option=com_docman\&view=download\&alias=15874rces003-14\&ltemid=30192

24. Lermen Junior N. Currículo Baseado em Competências para Medicina de Família e Comunidade. Sociedade Brasileira de Medicina de Família e Comunidade (SBMFC) [Internet]. 2015. [acesso 2017 Maio 2]. Disponível em: http://www.sbmfc. org.br/media/Curriculo\%20Baseado\%20em\%20Competencias(1).pdf

25. Moaveni A, Valin S, Hilderal V. Family and Communitty Medicine Clerkship. University of Toronto. [Internet]. 2014. [cited 2017 Maio 2]. Available from: http://dfcm.webservices.utoronto.ca/Assets/DFCM2+Digital+Assets/ Family+and+Community+Medicine/DFCM+Digital+Assets/Undergraduate/HPD+Handbook+14.15.pdf

26. Tandeter H, Carelli F, Timonen M, Javashvili G, Basak O, Wilm S, et al. A 'minimal core curriculum' for Family Medicine in undergraduate medical education: a European Delphi survey among EURACT representatives. Eur J Gen Pract. 2011;17(4):217-20. DOI: http://dx.doi.org/10.3109/13814788.2011.585635

27. Franco CAGS, Cubas MR, Franco RS. Currículo de medicina e as competências propostas pelas diretrizes curriculares. Rev Bras Educ Méd. 2014;38(2):221-30. DOI: http://dx.doi.org/10.1590/S0100-55022014000200009

\section{* Referências sugeridas para estudo de texto:}

GUSSO, Gustavo; LOPES, José Mauro Ceratti (Org.). Tratado de medicina de família e comunidade: princípios, formação e prática. Porto Alegre: Artmed, 2012.

DUNCAN, Bruce B. et al. Medicina ambulatorial: condutas de atenção primária baseada em evidências. 4. ed. Porto Alegre: ArtMed, 2013. 1952 p.

FERNANDES, Carmen Luiza Corrêa; CURRA, Lêda Chaves Dias. Ferramentas de Abordagem Familiar. PROMEF. Porto Alegre: ArtMed, 2005.

Cadernos de atenção básica. Disponíveis em: http://dab.saude.gov.br/portaldab/biblioteca.php?conteudo=publicacoes

Protocolos de encaminhamento da Atenção Básica para Atenção Especializada. Disponíveis em: http://dab.saude.gov.br/ portaldab/biblioteca.php?conteudo=publicacoes

a Departamento de Saúde Coletiva, Universidade Federal de Ciências da Saúde de Porto Alegre (UFCSPA). Porto Alegre, RS, Brasil. angelajben@gmail.com (Autora correspondente); jmauro.lopes@ @erra.com.br; carmend@ufcspa.edu.br, cgdaudt@hotmail.com; eugenia@ufcspa.edu.br, meugeniap2@gmail.com;

monica@ufcspa.edu.br 